\title{
A Comparative Study on Efficacy of Ciprofloxacin with Tobramycin Ophthalmic Solution in Acute Conjunctivitis
}

\section{Mayuri Gupta Pathak*}

Assistant Professor, Patan Academy of Health Sciences, Nepal

*Corresponding Author: Mayuri Gupta Pathak, Assistant Professor, Patan Academy of Health Sciences, Nepal.

Received: November 01, 2019; Published: November 05, 2019

DOI: 10.31080/ASPS.2019.03.0432

\begin{abstract}
Acute conjunctivitis (AC) is a common disease in both developing and industrialized countries. Conjunctivitis is an inflammation of the conjunctiva usually occurs as a result of infection or allergy, resulting in a so-called "red eye" or "pink eye." Acute conjunctivitis can be viral in nature; however, as many as $78 \%$ of cases in children and half of cases in adults are caused by bacteria. Worldwide, there are an estimated 5 million cases of neonatal infectious conjunctivitis per year. It is usually a benign, self-limited condition. Two drugs though from different groups, both exerting antimicrobial action and are extensively used in acute bacterial conjunctivitis in Nepal Medical College. It will therefore be of interest to know the difference between the efficacies of these two widely utilized drugs. Ciprofloxacin ophthalmic solution (fluoroquinolones) and Tobramycin ophthalmic solution (aminoglycosides), which are the most commonly used as topical antimicrobial agent in context of Nepal.
\end{abstract}

Keywords: Ciprofloxacin; Acute Conjunctivitis; Nepal

\section{Introduction}

- Defined as conjunctivitis with symptoms of less than 3-4 weeks duration, and is the eye disorder most commonly seen by a general practitioner

- Neisseria gonorrhoeae is the most frequent cause of hyperacute bacterial conjunctivitis.

- Acute conjunctivitis can be viral in nature; however, as many as $78 \%$ of cases in children and half of cases in adults are caused by bacteria.

- Worldwide, there are an estimated 5 million cases of neonatal infectious conjunctivitis per year.

- Various classes of antibiotics have been used including aminoglycosides, polymyxin B combinations, macrolides, sulfonamides in acute conjunctivitis

- The rational behind the use of either ciprofloxacin or tobramycin ophthalmic solution is; they are broad spectrum antimicrobials which are active against gram positive and gram negative bacteria.

- Two drugs though from different groups, both exerting antimicrobial action and are extensively used in acute bacterial conjunctivitis in Nepal Medical College.
- Thus, it is planned to compare the efficacy of two topical antimicrobial agents: ciprofloxacin ophthalmic solution and tobramycin ophthalmic solution, which are the most commonly used as topical antimicrobial agent in context of Nepal.

\section{Methodology}

- A prospective study was conducted from January 2018 to August 2019. Each of the patients was assigned with a serial number in terms of lottery system.

- $\quad$ All the patients were randomized into two groups: Group A and Group B.

- Group A patients received Ciprofloxacin ophthalmic solution.

- Group B patients received Tobramycin ophthalmic solution.

- In both groups, length of infected days, intended drug prescribed were recorded.

\section{Follow up of the cases}

- Follow up for mild, moderate and severe cases were done after 3 days, 5 days and 7 days.

- Acute bacterial conjunctivitis before and after treatment were determined using sign and symptoms scoring scales. 
- $\quad$ Sign and symptoms scoring scale consist of hyperemia, conjunctival discharge, congestion and bulbar conjunctival injection.

- $\quad$ The used sign and symptoms scale help to determined the different severity of disease (absent, mild, moderate and severe) and also scores (0, 1, 2 and 3). A response of 'none' is scored as ' 0 ', mild-1, moderate-2, severe-3.

- A decrease in the clinical signs and symptoms to a total severity score 0 or 1 will be considered as clinical cure.

\section{Statistical analysis}

- $\quad$ All the collected data were entered in and statistical analysis carried out using Statistical Package for Social Sciences (SPSS) version 20. The data was analyzed for age, sex and cure rates.

- Results were expressed as mean \pm standard deviation. Descriptive statistics like Chi-square test were used to analyze socio-demographic data.

- Different statistical test of significance like pair $t$ test and independent sample $t$ test were applied to find the $p$ value.

- Cure rates at the end of 7 days for mild cases were compared by using Non parametric Mcnemar test.

\section{Age and gender wise distribution of conjunctivitis} patients

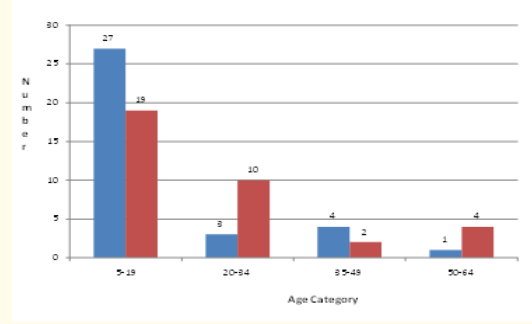

Figure 1

\section{Sign and symptoms score before and after treatment in ciprofloxacin group (Day 1)}

Treatment for mild, moderate and severe cases

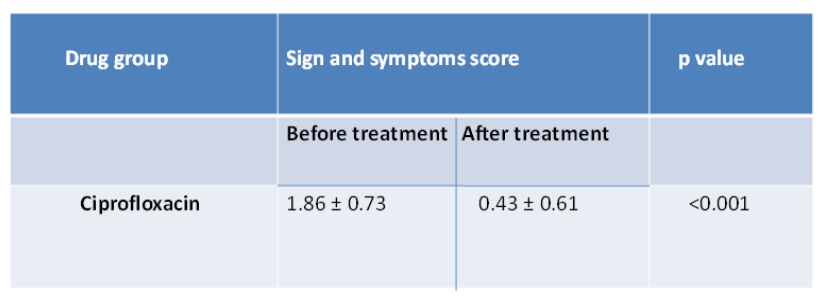

Figure 2
Sign and symptoms score before and after treatment in Tobramycin group (Day 1)

Treatment for mild, moderate and severe cases

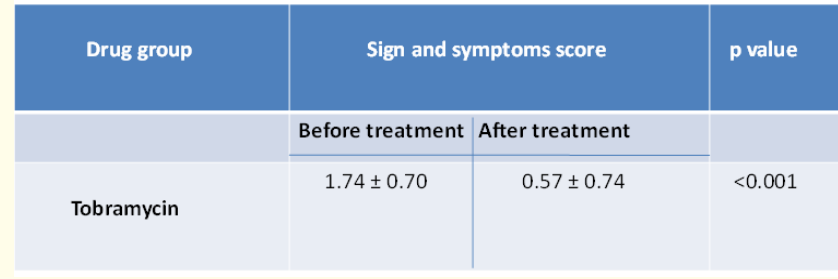

Figure 3

\section{Comparison of sign and symptoms in two drugs groups}

\begin{tabular}{|c|l|c|c|}
\hline \multirow{2}{*}{ Days of treatment } & \multicolumn{2}{|c|}{ Drug groups } & p value \\
\hline Day 1 & Ciprofloxacin & Tobramycin & \\
\hline Follow up & $1.86 \pm 0.73$ & $1.74 \pm 0.70$ & $>0.05$ \\
\hline & $0.46 \pm 0.61$ & $0.57 \pm 0.74$ & $>0.05$ \\
\hline
\end{tabular}

Figure 4

\section{Comparison of cure rates after 7 days in two drug groups (Mild)}

\begin{tabular}{|c|c|c|c|c|}
\hline Drug groups & $\begin{array}{l}\text { Total no. of } \\
\text { patients }\end{array}$ & \multicolumn{3}{|c|}{$\begin{array}{l}\text { After } \mathbf{7} \text { days } \\
\text { Cure rates }\end{array}$} \\
\hline \multirow{2}{*}{ Ciprofloxacin } & \multirow{2}{*}{12} & Cured no. & Uncured no. & $\begin{array}{l}\text { Cured } \\
\text { percentage }\end{array}$ \\
\hline & & 12 & 0 & 100 \\
\hline Tobramycin & 14 & 14 & 0 & 100 \\
\hline
\end{tabular}

Figure 5

Sign and symptoms score before and after treatment in ciprofloxacin and Tobramycin group (Moderate) (Mean sign and symptoms score on 5 th day as compared to $1^{\text {st }}$ )

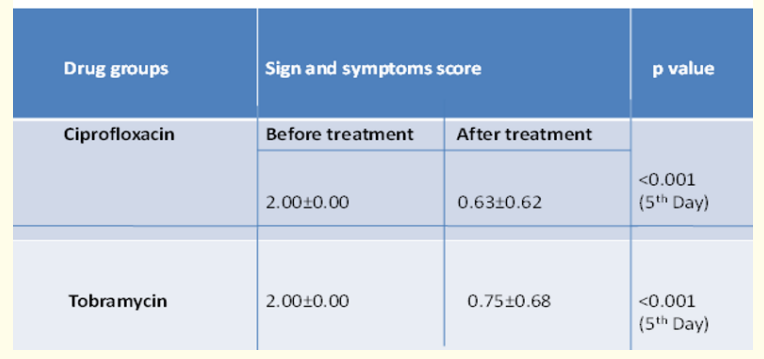

Figure 6 


\section{Sign and symptoms score before and after treatment in two drug groups (Severe) \\ (Mean sign and symptoms score on $3^{\text {rd }}$ day as compared to $1^{\text {st }}$ day)}

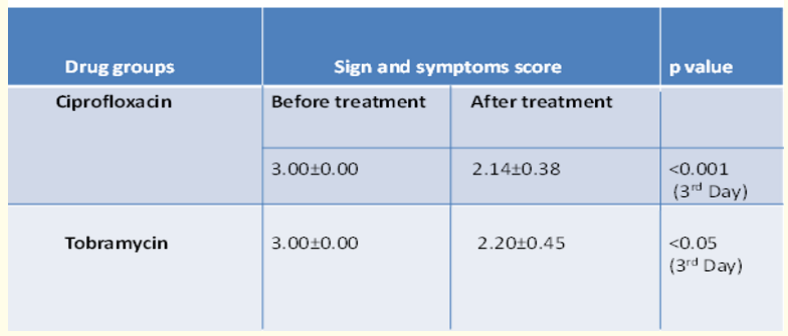

Figure 7

\section{Sign and symptoms score before and after treatment in two drug groups (Severe)}

Mean sign and symptoms score on 7 th day as compared to $1^{\text {st }}$ day

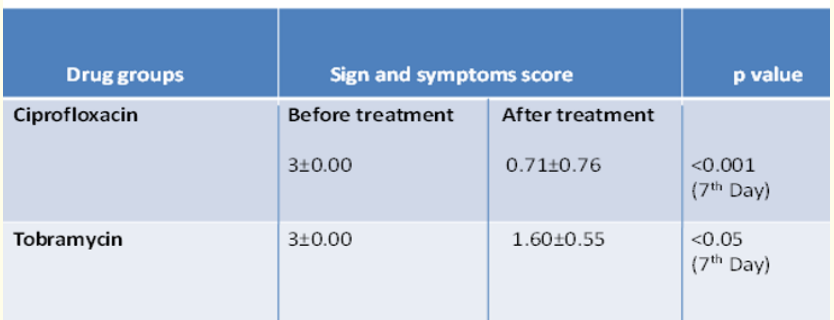

Figure 8

Gender wise sign and symptoms score after treatment in two drug groups

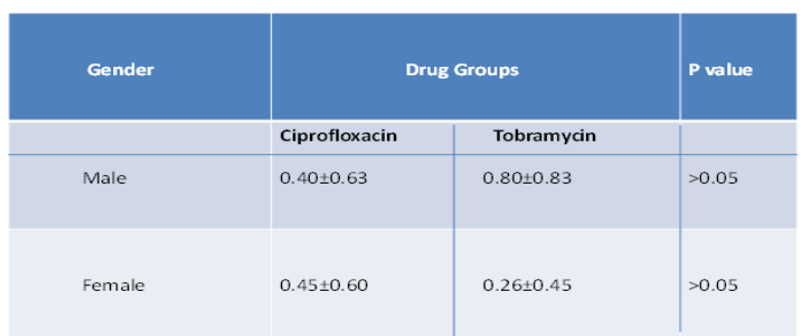

Figure 9

\section{Discussion}

- In the present study, comparative clinical efficacy of ciprofloxacin with tobramycin ophthalmic solution in acute conjunctivitis was attempted by using sign and symptoms score.

- This prospective study was conducted in Department of Ophthalmology, NMCTH, Jorpati, Kathmandu from January 2018 to August 2019.
- A total of 70 patients of either suffering from acute bacterial conjunctivitis were studied. The data obtained was statistically analyzed.

- The main objective of this study was to find the efficacy of two study drugs ciprofloxacin and tobramycin in acute bacterial conjunctivitis.

- However, socio demographic data collected was analyzed. In the present study, it is observed that male and female patients were equally affected.

- There was no statistically significant difference $(p>0.05)$ in gender wise distribution of acute conjunctivitis in two drug groups.

- In the study, it is observed that the number of acute bacterial conjunctivitis patients were higher in children of the age group 5-19 years (46 patients).

- The mean age of the patients was $19.63 \pm 15.49$ years (range 5-64 years)

- In the present study, the sign and symptoms scores are categorized as mild, moderate and severe according to severity of diseases.

- $\quad$ Follow up for mild, moderate and severe cases were done after 3 days, 5 days and 7 days. In mild cases, all 12 patients in ciprofloxacin and 14 patients in tobramycin were clinically cured at day 7.

- In moderate cases, out of 16 patients, 15 were cured in ciprofloxacin group whereas out of 16 patients, 14 were cured in tobramycin group at day 5 .

- The mean sign and symptoms score between two study drugs were compared; no statistically significant reduction ( $p>0.05$ ) was observed.

- In severe cases, out of 7 patients, 6 were cured in ciprofloxacin group whereas out of 5 patients, 2 were cured in tobramycin group at 7 days.

- At day 3 for severe patients, the mean sign and symptoms score between study drugs were compared; no statistically significant reduction ( $p>0.05)$ was observed.

- However, at day $7^{\text {th }}$ for same patients, the mean sign and symptoms score between two study drugs were compared; statistically significant reduction was observed $(\mathrm{p}<0.05)$

- This study showed that both study drugs were equally effective in mild cases. But in moderate and severe cases, ciprofloxacin is more effective than tobramycin.

- The results were equivocal as no such earlier studies were found [1-15]. 


\section{Conclusion}

- It was a prospective, randomized hospital based study and the efficacy of drugs under study was evaluated by using cure rates in both groups of treatment

- The result of this study indicates that acute bacterial conjunctivitis is more common in children than adults.

- $\quad$ Male and female were equally affected.

- Both study drugs were effective in the treatment of acute bacterial conjunctivitis

But the cure rates were significantly higher in ciprofloxacin group as compared to tobramycin group

Limitation

- In this study, sample size was small. Therefore, multicenter studies with large sample size are required.

\section{Recommendation}

- This study was not designed to find the association of sociodemographic factors with acute bacterial conjunctivitis.

- $\quad$ Studies are required to find the association. This study was not based on microbiological cure rates.

- So, further studies are warranted to assess both clinical and microbiological cure rates.

- This study comprises the patients suffering from acute bacterial conjunctivitis.

However, patients of viral conjunctivitis can be taken into consideration in other studies.

\section{Bibliography}

1. Richards A and Guzman-Cottrill JA. "Conjunctivitis". Pediatric Review 31.5 (2010): 196-208

2. Gigliotti F. "Acute conjunctivitis". Pediatric Review 16.6 (1995): 203-207.

3. Morrow GL and Abbott RL. "Conjunctivitis". American Family Physician 57 (1998): 735-746.

4. Hovding G. "Acute bacterial conjunctivitis". Acta Ophthalmologica 86 (2008): 5-17.

5. Cochereau I., et al. "3-Day Treatment With Azithromycin 1.5\% Eye Drops Versus 7-Day Treatment With Tobramycin $0.3 \%$ for Purulent Bacterial Conjunctivitis: Multicentre, Randomised and Controlled Trial in Adults and Children". British Journal of Ophthalmology 91 (2007): 465-469.

6. Sheikh A and Hurwitz B. "Antibiotics versus placebo for acute bacterial conjunctivitis (review)". Cochrane Database System Review 2 (2006): 1-18.
7. Diamant JI and Hwang DG. "Therapy for bacterial conjunctivitis". Ophthalmology Clinics of North America 12 (1999): 15-20.

8. Everitt $\mathrm{H}$ and Little P. "How do GPs diagnose and manage acute infective conjunctivitis? A GP survey". Family Practice 19 (2002): 658-660.

9. McDonnell PJ. "How do general practitioners manage eye disease in the community?" British Journal of Ophthalmology 72 (1988): 733-736.

10. Karpecki P., et al. "Limitations of current antibiotics for the treatment of bacterial conjunctivitis". Optometry and Vision Science 10 (2010): 1097.

11. Gigliotti F., et al. "Efficacy of topical antibiotic therapy in acute conjunctivitis in children". Journal of Pediatrics 104 (1983): 623-626.

12. Moroi SE and Lichter PR. "Ocular Pharmacology". In: Hardman JG, Limbird LE, Molinoff PB, Ruddon RW, Gilman AG, editors. Goodman and Gilman's Pharmacological Basis of Therapeutics. 9th ed. New York: McGraw-Hill 1996: 1630.

13. Hyndiuk RA., et al. "Comparison of ciprofloxacin ophthalmic solution $0.3 \%$ to fortified tobramycin-cefazolin in treating bacterial corneal ulcers: Ciprofloxacin Bacterial Keratitis Study Group". Ophthalmology 103 (1996): 1854-1862.

14. Oliver GF., et al. "Acute infective conjunctivitis: evidence review and management advice for New Zealand practitioners". New Zealand Medical Association 122 (2009): 69-71.

15. Sheikh A., et al. "Antibiotics versus placebo for acute bacterial conjunctivitis". Cochrane Database System Review 2 (2000): 1211.

Volume 3 Issue 12 December 2019

(C) All rights are reserved by Mayuri Gupta Pathak. 